club, was 678 steps $(308,1048)$ in favour of the intervention $(\mathrm{p}<0.001)$; the mean difference in time spent sitting was $1.7 \mathrm{~min}(-10.4,13.8) \quad(p=0.78)$. We also found significicant improvements in self-reported food intake, weight, resting blood pressure and some cardio-metabolic blood biomakers in favour of the intervention. Seven serious adverse events were reported. Of these, 5 were assessed as likely to be associated with EuroFIT participation.

Discussion Participation in EuroFIT led to modest improvement in physical activity but not sedentary time at 12 months. Public health messages to be more physically active and eat well are now widely understood but the 'sit less' message is newer and less is known about how to achieve it. In this context coaches and participants may have found it difficult to disentangle advice about sitting less from advice about being more physical activity. Differences in outcomes between FFIT and EuroFIT will be discussed.

Authorship is on behalf of the EuroFIT consortium.

\section{OP81 APPLYING HURDLE MODELS TO ESTIMATE SOCIOECONOMIC INEQUALITIES IN MODERATE-TO- VIGOROUS PHYSICAL ACTIVITY: ANALYSIS USING THE HEALTH SURVEYS FOR ENGLAND 2008 AND 2012}

S Scholes*, JS Mindell. Epidemiology and Public Health, University College London, London, UK

\subsection{6/jech-2018-SSMabstracts.80}

Background Guidelines recommend adults engage in at least $150 \mathrm{~min}$ of moderate-to-vigorous physical activity (MVPA) per week. It is unknown whether describing socioeconomic inequalities using the average amount of time persons spend in MVPA masks: (1) disparities in the proportion of persons that are active, or (2) disparities in the amount of time that persons who are active spend engaging in physical activity.

Methods Hurdle models are a new way of accommodating continuous physical activity data with: (1) an excessive amount of zeros (non-participation), and (2) a continuous positivelyskewed part (the amount of time active persons spend being active). Using the Health Surveys for England $(n=16,012$; HSE 2008; 2012), we applied hurdle models to estimate inequalities in these two separate parts of MVPA data, and assess changes over time. Analyses were sex-specific and adjusted for body mass index and smoking. Separate analyses were performed for overall MVPA and for five activity domains, including walking and sports/exercise.

Results are presented as Marginal Effects (ME) with 95\% Confidence Intervals (95\% CIs). The MEs represent absolute differences between the highest- and lowest-income groups in: (1) the percentage of participants who were active, and (2) the average hours-per-week (hpw) spent in MVPA conditional on participants being active (i.e. hpw being greater than zero). Results The proportion of participants who performed any activity was highest in the highest-income group. The $\mathrm{ME}$ for overall MPVA was 12.5 percentage points (pp) [95\% CI 10.3 to $14.7 \mathrm{pp}$ ] in men; $11.6 \mathrm{pp}$ [9.5-13.7 pp] in women. Similar patterns were found for walking [men: $19.8 \mathrm{pp}$ : 16.7-22.8 pp; women: $15.0 \mathrm{pp}$ : $12.4-17.6 \mathrm{pp}]$ and for sports/exercise [men: 20.0 pp: 16.9-23.2 pp; women: 23.1 pp: 20.4-25.9 pp].

Differences in the amount of time spent in overall MVPA (amongst those doing any) also favoured high-income participants [men: 3.5 hpw: 2.4-4.7 hpw; women: 3.3 hpw: 2.5-4.2 hpw]. High-income participants spent on average 1 hpw more doing sports/exercise [men: 0.9 hpw: 0.0-1.8 hpw; women: $1.2 \mathrm{hpw}$ : 0.7-1.7 hpw]. However, time spent walking (amongst those doing any) showed the opposite pattern in men being $1.9 \mathrm{hpw}$ lower for those in the highest-income group [ -2.8 to $-0.9 \mathrm{hpw}$. Patterns were similar in 2008 and 2012. Findings were robust to different model specifications (e.g. using two-part models).

Conclusion Inequalities in overall MVPA and in sports/exercise were pronounced for the hurdle of participation and for the amount of time spent being active. For walking among men, inequalities were sharpest for the hurdle of participation, highlighting the importance of interventions designed to increase walking among inactive low-income individuals. Our results will be updated when HSE 2016 data are available (spring 2018).

\section{Health ageing}

\section{OP82 \#POPULATION PRIORITIES FOR SUCCESSFUL AGEING: A RANDOMISED VIGNETTE EXPERIMENT}

${ }^{1}$ E Whitley*, ${ }^{2} \mathrm{M}$ Benzeval, ${ }^{1} \mathrm{~F}$ Popham. ${ }^{1} \mathrm{MRC} / \mathrm{CSO}$ Social and Public Health Sciences Unit, University of Glasgow, Glasgow, UK; ${ }^{2}$ Institute for Social and Economic Research, University of Essex, Colchester, UK

\subsection{6/jech-2018-SSMabstracts.81}

Background Worldwide population ageing has resulted in a growing interest in 'successful ageing' but there is no established consensus as to what this entails. Existing evidence is largely qualitative, confounded, and restricted to older ages. We aimed to provide robust, unconfounded estimates of the relative importance placed by the general population on six commonly-used dimensions of successful ageing (disease, disability, physical functioning, cognitive functioning, interpersonal engagement, and productive engagement).

Methods We conducted a randomised experiment in wave 9 of the Understanding Society Innovation Panel, a stratified, geographically clustered sample of 2545 men and women designed to be representative of the British population. A total of 2010 (79\%) respondents took part in the experiment and were presented with three vignettes, each describing a hypothetical 75 year old person with randomly determined favourable or unfavourable outcomes for each of the six dimensions. Respondents were asked how successfully the person described was ageing on a scale from 0 (not successfully) to 10 (very successfully).

Results The main outcome measure for each of the dimensions was the difference in mean scores comparing vignettes with favourable versus unfavourable attributes; as each dimension was presented in the same way, direct comparisons can be made between them to understand their relative importance. Scores were allocated to 5967 vignettes and those in which dimensions were favourable were allocated higher scores than those in which they were unfavourable. However, the relative importance given to each dimension varied. Across all participants, the largest differences were observed for cognitive function (difference $(95 \% \mathrm{CI}): 1.20(1.11,1.30)$ ) and disability $(1.18(1.08,1.27))$ and the smallest for disease $(0.73(0.64$, $0.82))$ and productive engagement $(0.58(0.49,0.66)$. Differences for physical functioning and interpersonal engagement 